ONCOLOGY; PERIODONTOLOGY

\section{Gingival squamous cell carcinoma: diagnostic delay or rapid invasion?}

Seoane J, Varela-Centelles PI et al. J Periodontol 2006; 77: 1229-1233

Early invasion of bone suggests that suspect gingival lesions require rapid attention.

Gingival cancer can resemble common periodontal lesions, and this may lead to diagnostic delay. In a Spanish hospital over a 4 yr period, 59 consecutive cases of primary intra-oral squamous cell carcinoma were identified in patients of mean age 61 yrs.

Twelve of these tumours were gingival, and 9 were diagnosed within 1.5 months. Fifteen of the 46 other tumours were diagnosed within the same period. All but 3 non-gingival, and all gingival, tumours were diagnosed within $1 \mathrm{yr}$.

However, the gingival tumours were significantly more advanced in tumour stage. Eight of the 12 were at stage 4, but only 14 of the 47 non-gingival tumours $(P<0.05)$. The authors discuss their findings and suggest that lesions which might be gingival tumours require special attention because of their early invasion of bone.

doi:10.1038/sj.bdj.4814264

CARDIOVASCULAR DISEASES; PERIODONTOLOGY

\section{Periodontal pathogens in atheromatous plaques isolated from patients with chronic periodontitis}

Padilla C, Lobos 0 et al. J Periodont Res 2006; 41:350-353

Some periodontal bacteria were identified in pockets and atheroma specimens from the same patients.

Studies have suggested possible links between cardiovascular and periodontal diseases. Periodontal examination with microbial sampling was performed in 12 patients one week prior to endarterectomy and examination of atheromatous plaques.

Using PCR, $P$. gingivalis was identified in 7 periodontal isolates, A. actinomycetemcomitans in $7, P$. intermedia in 5 and $T$. forsythia in 2. Three samples yielded none of these organisms. A. actinomycetemcomitans alone was found in 2 atheromatous plaques, both of which came from patients with the organism in periodontal isolates.

The authors discuss these findings, but in a cross-sectional study are unable to show whether the organisms colonised arteries before or after atheroma developed, or whether the organisms have any cardiovascular health significance.

doi:10.1038/sj.bdj.4814266

\section{ORAL PATHOLOGY}

\section{Clinical pathologic aspects of oral leukoplakia in smokers and nonsmokers}

Freitas MD, Blanco-Carrión A et al. OralSurg 2006; 102: 199-203

Leukoplakia in nonsmokers may be a more significant lesion.

Studies have suggested that leukoplakia is commoner in smokers, but is often less likely to lead to serious complications than in nonsmokers. In this retrospective study, 73 consecutive cases were considered, but 21 had inadequate smoking data. Of the remainder, 41 came from smokers and 11 from nonsmokers. Full biopsy data were available for 28 patients.

Twenty of the lesions in smokers were from the buccal mucosa or floor of the mouth, while in nonsmokers, 7 were from the tongue. While the statistics in this paper appear to be inaccurately stated, with 3 odds ratios apparently outside the range of their confidence intervals, the raw data are interesting. In 8 nonsmoker biopsies, there were 3 lesions with epithelial dysplasia, while only one such lesion was found in 20 nonsmoker biopsies.

doi:10.1038/sj.bdj.4814265

ORTHODONTICS; ORAL SURGERY; BEHAVIOURAL SCIENCE

Psychological support for orthognathic patients - what do orthodontists want?

Juggins KJ, Feinmann C etal. JOrthod 2006; 33: 107-115

Most orthodontists involved in orthognathic surgery perceived a personal need for training in this area.

Patients with dentofacial deformity may be more likely to experience psychological distress. Studies suggest that those who undergo orthognathic treatment are usually well-adjusted individuals. However, evidence suggests that $5-8 \%$ of such patients are dissatisfied after treatment. This study sought the opinions of consultant orthodontists on psychiatric or psychological referral before orthognathic treatment. Questionnaires were sent to all 245 registered consultant orthodontists in the UK, and 183 replied.

About 90\% of orthodontists thought that some of their patients would benefit from referral for psychological assessment, and over $12 \%$ of these thought all their patients would benefit. However, when asked how many patients they actually referred, $41 \%$ did this for about 10\% of patients, and $60 \%$ made no referrals. When asked about their personal use of psychological assessment for their patients, only $7 \%$ did this, $81 \%$ had had no training and $85 \%$ felt that they would benefit from such training.

doi:10.1038/sj.bdj.4814267 